\title{
RNA Interference (RNAi) as a metronome of the circadian cadence
}

\section{Utpal Bhadra ${ }^{1 *}$, Paromita Das ${ }^{2}$ and Manika Pal-Bhadra ${ }^{2}$}

${ }^{1}$ Functional Genomics and Gene Silencing Group, Centre for Cellular and Molecular Biology, Hyderabad-500007, India

${ }^{2}$ Centre for Chemical Biology, Indian Institute of Chemical Technology, Hyderabad-500007, India

\begin{abstract}
The circadian clock of an organism is intricately entwined between a multitude of intercellular and intracellular networks consolidating the physiological activities into a refined rhythmic pattern. The key to these rhythms lie in their exceptional competence in either self-sustaining or in resetting themselves to the most suitable stimuli, neglecting the irrelevant ones. Apart from the prevalent notion about the dominant role of transcription and translation in the clock regulation, recent findings have unfurled the importance of post-transcription and post-translation in modulating the same. This review aims at evolving a new player in clock control via the incredible phenomena of RNA interference (RNAi), which is executed mainly through the microRNAs and the long non-coding RNAs. It enlightens us with replete evidences that effectively establish the cryptic yet enigmatic role of different core components of RNA interference and their machinery in the modulation of all the unexplored facets for the sustenance and constancy of the biological rhythm.
\end{abstract}

Keywords: Circadian oscillation; RNA interference; Micro RNA; Long non-coding RNA; Biological clock

Abbreviations: PER: Period; TIM: Timeless; CLK: Clock; CCA1: Circadian and Clock Associated 1; LHY: Late Elongated Hypocotyl; TOC1: Timing of CAB Expression 1; PRR: Pseudo Response Regulator; CRY: Cryptochrome; RISC: RNA Induced Silencing Complex; JET: Jetlag; DBT: Double time; CK: Casein Kinase; SGG: Shaggy; PP2a: Protein Phosphatase 2a; PP1: Protein phosphatase; GSK3: Glycogen Synthase Kinase 3; ZT: Zeitgeber; VRI: Vrille

\section{Introduction}

The Earth's axial rotation and solar revolution bring out anticipated changes in light and temperature. Different zones of the earth are subjected to a widely varying temperature and light gradient according to their geographical location. To acclimatize the organism's physiological processes in response to these abiotic factors, natural selection has favoured and established the internal timekeeping rhythm in the organisms, which is referred to as the circadian rhythm. The circadian rhythm machinery therefore not only acts a daily executor of physiological processes but it also acts an attendant to adapt with the changing seasons [1].

After the discovery of RNAi, a sea change incentral dogma has been arrived at, ever since the identification of the reverse transcriptase. Different proteins are involved in maintenance of the circadian rhythm and their expression is finely tuned. This tuning is mediated by the delayed translation, rapid degradation and phosphorylation mediated changes in protein function, light dependent regulation etc. Along with these mechanisms, the non-coding RNA mediated regulation adds a new level of check on this physiological phenomenon. This rhythmic behaviour is present in different organisms across the globe. The proteins involved in maintenance of this rhythm are different in different organisms. From plants of lower order to animals of higher order, circadian rhythm are used to better acclimatize themselves to environment.

The sequencing of the Arabidopsis genome has led to the identification of different orthologous of the recognized clock proteins. The Arabidopsis clock is unique bearing conserved feedback loops consisting of three interlocked feedback loops, with two single Myb domain transcription factors, Circadian and Clock Associated1 (CCA1) and Late Elongated Hypocotyl (LHY). These factors control the positive regulation of three TOC1 relatives, PRR5, PRR7 and PRR9. TOC1 is a clock component that controls the oscillation of the mRNA and the protein protein produced there of, CCA1/LHY is negatively regulated by PRR5/7/9. CCA1 and LHY is transcriptionally repressed post degradation by ZTL to regulate the titre of TOC 1 which primarily determines the circadian period [2].

The circadian clock in mammals has their separate counterparts in Drosophila, with some variations in the circadian loop mechanism. CLK and BMAL1 are the heterodimers that act as transcriptional activators for several light-induced promoters and turn on three PER genes, two CRY genes, coding for cryptochromes. The PER and CRY proteins produced from the respective genes, get exported to nucleus posttranslation. Subsequently, these proteins prevent the transcription of PER and CRY through the inhibition of CLOCK-BMAL1 and soon after their degradation, the PER and CRY genes are re-transcribed by the transcriptional activity of CLOCK and BMAL1. Thus, there are negative feedback loops that lead to the reverse phase oscillation of BMAL1 and PER/CRY.

\section{Modern view of circadian rhythm}

The circadian rhythm is controlled by different proteins in different organisms. However, due to the limited scope of this review, we shall solely focus extensively on the circadian proteins, involved in Drosophila. The Drosophila physiology and behaviour comprises multiple aspects like eclosion [3], olfactory sensitivity [4], egg laying [5], courtship [6,7], gustatory sensitivity [8] or learning and memory [9] which are restricted to persist in response to particular environmental conditions. Presence of a sophisticated molecular, genetic and biochemical tools to study complex fly behaviour along with the clarity of Drosophila's neuronal network organization with 150 clock cells per brain hemisphere makes Drosophila a promising and reliable model for circadian studies. Particularly in the case of eclosion, where adult flies emerge from their pupal cases during the cool and moist morning or dusk, with folded wings, to avert the risk of desiccation and later the

${ }^{*}$ Corresponding author: Utpal Bhadra, Functional Genomics and Gene Silencing Group, Centre for Cellular and Molecular Biology, Uppal Road, Hyderabad India-50007, Tel: 91-40-27192513; Fax: 91-40-27160591, E-mail: utpal@ccmb.res.in

Received June 17, 2016; Accepted June 21, 2016; Published June 28, 2016

Citation: Bhadra U, Das P, Pal-Bhadra M (2016) RNA Interference (RNAi) as a metronome of the circadian cadence. Mol Biol 5: 166. doi:10.4172/21689547.1000166

Copyright: $\odot 2016$ Bhadra U, et al. This is an open-access article distributed unde the terms of the Creative Commons Attribution License, which permits unrestricted use, distribution, and reproduction in any medium, provided the original author and source are credited. 
wings open after the cuticle hardens. The pupae that were exposed to $12 \mathrm{~h}$ light: $12 \mathrm{~h}$ dark cycle, with constant darkness, were able to maintain the time of eclosion to a subjective dawn probably because of an internal pacemaker [10]. Even post-emergence, the adult flies restrict their flight, foraging and mating activities to the day time and are relatively passive to stimulus, exhibiting a state of rest $[11,12]$ during the night. Multiple tissues and cells coordinate their activities with these external cues to give rise to a precise regulation of the circadian behaviour.

The first "clock gene" to be discovered was period or per, using a phenotype-based screening of flies that were exposed to mutagens. Three period mutants, long $\left(\mathrm{PER}^{\mathrm{L}}\right)$, short $\left(\mathrm{PER}^{\mathrm{S}}\right)$ and arrhythmic $\left(\mathrm{PER}^{0}\right)$, isolated for the same gene were found to be involved in rhythmic eclosion $[3,13,14]$. The PER protein sequence failed to reveal much about its participation in the clock due to the presence of a stretch of threonine-glycine repeats responsible for acclimatizing organisms to a variety of thermal conditions [15]. A region same as that of the Drosophila Single-Minded (SIM) and mammalian aryl hydrocarbon receptor nuclear translocator (Arnt) proteins, was found to help in protein-protein interaction which was known as the Per-Arnt-Sim (PAS) domain [16-18].

The cyclical expression of mRNA and protein of PER gene suggested that PER protein is required for circadian time-keeping via a feedback loop $[19,20]$. Further studies revealed that PER gene is involved in feedback loop as a transcriptional repressor [21,22]. Besides, PER, which is crucial for the feedback regulation, along with other feedback loops constitute the "circadian feedback loops."

In the PER feedback loop, the PER gene is transcribed initially during the mid-day, leading to subsequently rising levels of PER mRNA till early evening. As the levels of PER continue to accumulate, it represses the transcription of PER. Hence, there is a negative feedback regulation of the level of PER transcripts. The remnant PER protein is eliminated on the next morning to initiate a new cycle of PER transcription. A combinatorial approach comprising genetic screens, molecular interaction assays, PER promoter analysis, and molecular searches for clock gene ortholog has unleashed additional clock genes like timeless (TIM), clock (CLK), cycle (CYC), double time (DBT), shaggy (SGG), casein kinase 2 (CK2) subunits, and cryptochrome (CRY) [16,23-31].

A screen for interacting partners of PER led to the discovery of TIM [32], which binds to the PAS domain of PER. A transcription regulatory elements called canonical E-box element (in this case 5'-CACGTG-3') in the per promoter activates per transcription and has been a conserved in "circadian" circuitry [33]. In mouse, genetic screens have identified clock genes CLK [34-36] that is a transcriptional activator. It is a member of the basic-helix-loop helix-PAS (bHLH-PAS) transcription factor family that binds to the E-box elements to activate transcription. Protein coding sequences from mouse CLK were used further to retrievethe the Drosophila CLK ortholog using a cDNA library screen [37].

In the core feedback loop, PER and TIM are found to be actively transcribed from ZT4 to ZT18 (ZT0 is lights on and ZT12 is lights off) provided CLK and its heterodimers CYC bind to the E-boxes of the PER and TIM promoters $[24,25,33,37]$. It is only $6-8 \mathrm{hrs}$ after this, that the PER and TIM proteins begin to amass in the cytoplasm followed by nuclear localization of PER-DBT and TIM by the phosphorylated PER. Soon after this, the nuclear complex promotes phosphorylation after binding to CLK, with transcriptional repression. Once that the lights are lit, the protein TIM gets degraded (Figure 1). Repeated
PER phosphorylation leads to the binding E3 ubiquitin ligase, SLIMB which channelizes the proteosomal degradation of PER. This make CLK hyper-phosophorylated and subsequently accumulated to initiate another new cycle of PER and TIM transcription [16,28-30]. The low levels of CLK mRNA in PER mutant and TIM mutant flies suggested that besides PER and TIM, CLK somehow activates transcription $[22,23,38]$.

A second "CLK feedback loop" comprising the transcription factors Vrille (VRI) and PAR domain protein $1 \varepsilon$ and $\delta(\operatorname{Pdp} 1 \varepsilon / \delta)$ was found to be interlinked with the core feedback loop wherein, the CLK loop, CLK-CYC binds E-boxes activating VRI transcription between -ZT4 and ZT16. With the rising VRI levels, VRI binds to the VRI/PDP1boxes (V/P-boxes) in the CLK promoter, resulting in the repression of

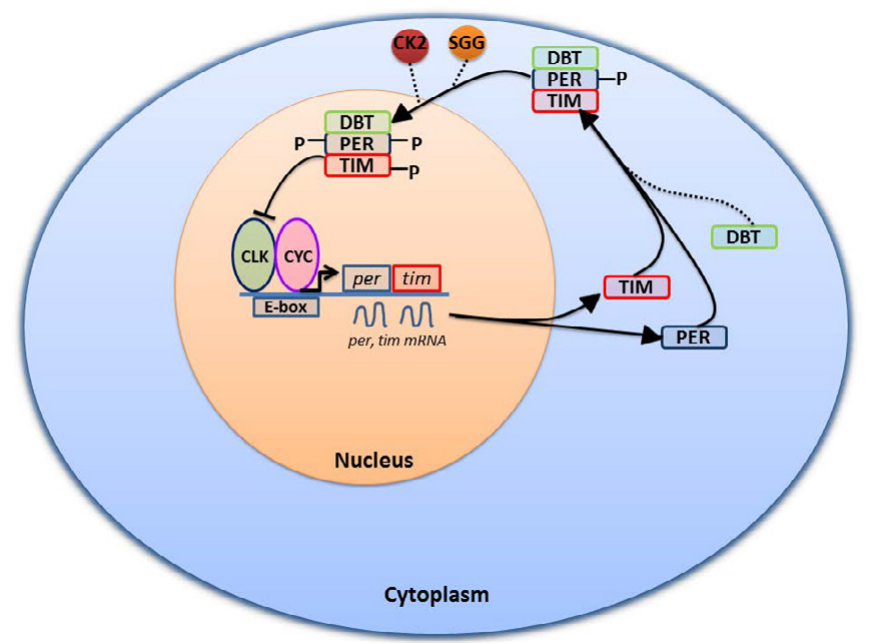

Figure 1: Modern view of circadian rhythm. (PER-TIM Cycle: After activation of the E-box by CYC-CLK heterodimer, PER and TIM gets transcribed and translated. Due to presence of TIM, degradation PER is delayed. DBT, kinase complexes with PER-TIM to form PER-TIM-DBT complex and the complex enters into the nucleus and inhibits CYC-CLK heterodimer to activate PER, TIM. Phosphorylation of PER by CK2 and TIM by SGG promotes nuclear localization of PER-TIM-DBT complex).

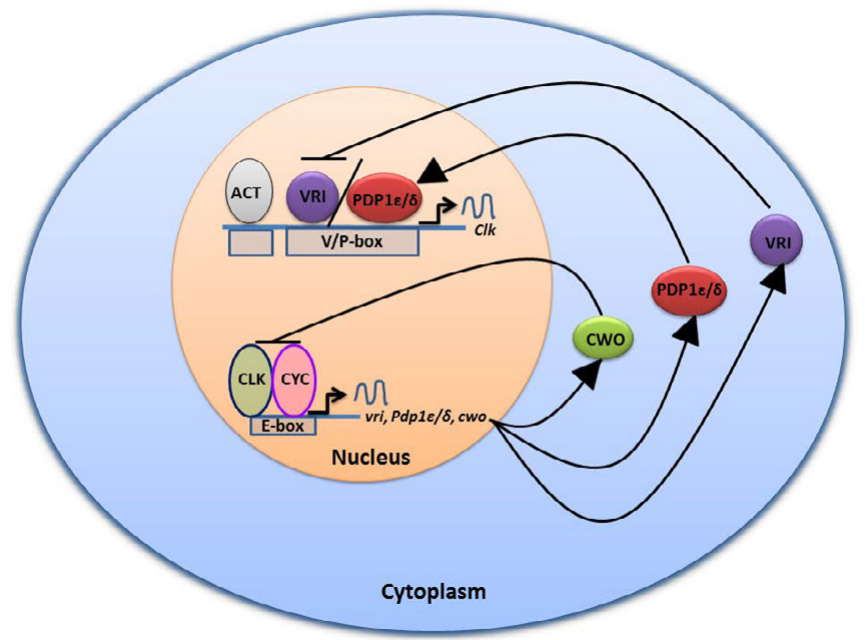

Figure 2: CLK feedback loop (Regulation of CLOCK: Along with the activation of PER and TIM, CYC-CLK heterodimer activates the transcription of CWO, VRI,

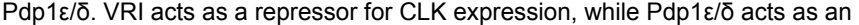
activator of clock gene). 
CLK transcription (Figure 2) [39-42]. Similar to PER-DBT and PERTIM-DBT VRI mRNA participates in the feedback inhibition lead to a protein decline. Soon after degradation of the PER complexes in the mid-day, the next cycle of VRI transcription and VRI-dependent repression ensues [16].

An alternate feedback loop, under the control of the bHLH-orange transcriptional inhibitor Clockwork Orange (CWO) [43-46] operates where CWO transcription is activated, and finally, CWO sends inhibitory signals to cease its own production in presence of the CLK-CYCmediated transcription. There is a delay of several hours between the PER phosphorylation and degradation, because of DBT phosphorylation at be PER serine 47 (S47) which produce typical specific SLIMB binding site [47]. Co-expression of DBT with PER in S2 cells, leads to DBT phosphorylation PER at multiple sites with final phosphorylation event on S47 [47-49]. Phosphorylation of PER by NEMO (NMO) at S596 is found to delay the phosphorylation of S47 by DBT, and thus PER degradation, consistent with a delay in PER degradation in the morning when NMO is over-expressed $[16,50,51]$. A model showing PER phosphorylation by $\mathrm{NMO}$ and DBT led to an alteration in the conformation of PER, which inhibited the phosphorylation of sites for SLIMB binding and further delayed the degradation of PER [50].

PER upon accumulation, gets phosphorylated and enters the nucleus, where TIM stabilizes it, by inhibiting phosphorylation at sites promoting SLIMB binding and degradation (Figure 3) [52]. PER is also dephosphorylated by PP2a, but not mediated by TIM [52,53]. PER, upon phosphorylation represses transcription [54], which shows that the TIM-based phosphorylation of PER represses CLK-CYC transcription. Experiments suggest that DBT is required for CLK phosphorylation [55-57] which was not in case of PER repression involving other kinases one of which is NMO [16].

TIM levels decline within $30 \mathrm{~min}$ of exposure to light, which destabilize PER by phosphorylation by a yet un-identified tyrosine kinase [58]. A screen for mutants that disrupted rhythms has identified CRYPTOCHROME (CRY) [31,59] an ortholog of blue light photoreceptor cryptochromes in plants $[60,61]$ whose elimination disrupts the core feedback loop in all except a few clock neurons. Direct binding of CRY to TIM leads to the proteasomal degradation of TIM in a light-dependent manner. However, the degradation of CRY occurs

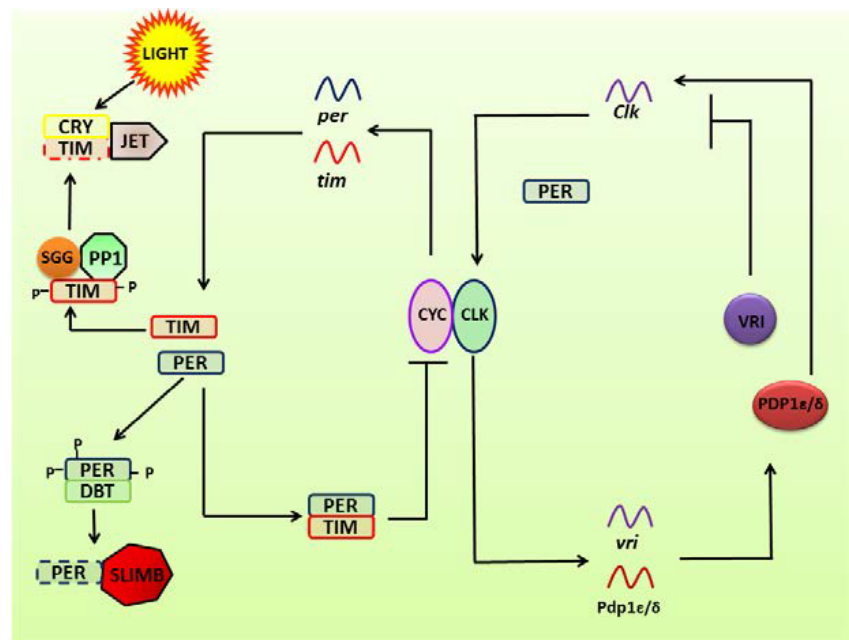

Figure 3: Circadian oscillation and protein phosphorylation (The proteins involved in circadian rhythm: Phosphorylation of PER by SLIMB leads to the proteasomal degradation of PER. Light dependent degradation of TIM and CRY by F-box protein, JET). more slowly than that of TIM [16].

The light-dependent degradation of both TIM and CRY is carried out by the F-box protein, Jetlag (JET) [62-64]. However, they are degraded sequentially due to a higher affinity of JET for TIM than CRY. Thus, CRY upon activation by light, becomes a substrate for JET binding, and it's binding, in turn makes TIM a higher affinity substrate for JET compared to CRY (Figure 3) [64]. COP9 signalosome is needed for the light induced degradation of TIM which acts downstream of JET, to aid the proteosomal degradation of TIM [16].

\section{Circadian oscillation and protein phosphorylation}

The PER and CRY protein is critical for the generation of a selfsustained circadian oscillation. However, there is often a time delay of several hours between the synthesis of PER and CRY proteins for their nuclear expression and the action of their inhibitors. This can be accounted to several mechanisms which alter the abundance, localization, and activity of transcriptional inhibitors by regulating the transcription, translation, and post-translational modifications of important components of the circadian oscillator. Of the other posttranslational modifications in eukaryotes, the most important ones are the phosphorylation of serine, threonine, and tyrosine residues. Such phosphorylation's serve as molecular switches between active and inactive protein states either by regulating the activity through kinases or indirectly by altering sub-cellular localization through transcription factors. Phosphorylation is also linked to protein degradation by the proteasome which occurs when the target proteins bearing specific motifs get phosphorylated, forming a recognition sequence for the ubiquitin-ligase complex.

\section{Intracellular Network Effects}

The prime mark of a circadian rhythm lies in its ability to reset itself to the most suitable stimuli, ignoring the irrelevant ones. The networks leading to a reliable circadian rhythmicity is divided into intracellular and intercellular networks. Intracellular processes comprise the cellular machinery regulating the constitution of the genes. These factors consists of a set of transcriptional and post-transcriptional mechanisms that control orderly circadian rhythm such as the core clock genes as the E-box activators, Clock and Bmall, and their transcriptional repressors, the Period and Cryptochrome genes. Recent findings have also included factors, Rev-erb-alpha and Rora that regulate the titre of the genes, Clock and Bmal1 [65].

\section{inter cellular communication}

Intercellular networks comprise those signalling pathways initiated from a particular circadian cell which influence rhythm in another. The vasoactive intestinal polypeptide (VIP) is considered as a signal for synchronising and fine-tuning the SCN cells. VIP is secreted in a rhythmic way from approximately $10 \%$ of neurons of the Supra Chiasmatic Nuclei [58]. Loss of VIP receptors in mice cause them to entrain poorly on a dim lit day, with one hour of light at dawn and another at dusk [59]. In the period of constant darkness, the locomotor activity of these mice loses rhythmicity in their locomotion and show a number of circadian periods [59-61]. Mice deficient in VIP signalling show a reduced free running period in behaviour and in the SCN [5961]. Other signals like the other neuropeptides like gastrin releasing peptide or vasopressin communicate via the gap junctions. Cues such as glucocorticoids or body temperature, control the SCN rhythmicity by acting in an indirect or direct way against the loss of VIP [6264]. An alternate approach to enhance the robustness is through the transcriptional amplification certain crucial clock genes. In this light, 
VIP raises cAMP [65-67], using (PKA) to phase advance the rate of firing in the SCN [68]. In this way, PER1 and PER2 can be induced for transcription depending upon the presence of CREB [69]. Certain transmitters, hormones, gap junctions and temperature fluctuations in the body entrain and increase the transcription of PER gene in the cell populations, thereby modulating the circadian rhythm [65].

\section{Role of rna interference in circadian rhythm, beyond transcriptional regulation}

In spite of the fact that the feedback loop of transcription-translation is common to all the current models of circadian oscillations, clock modulation is supposed to be highly dependent on the post-transcriptional and post-translational mechanisms. In some of the cases, an alternative the cyano bacterial clock is under the regulation of a three-protein (KaiA, $\mathrm{KaiB}$ and $\mathrm{KaiC}$ ) system that rhythmically oscillates in phosphorylating KaiC [66]. In flies and mammals, clock protein phosphorylations regulate their turnover rate and their interactions with other proteins and subcellular localization and in this way, are able to control the speed of the clock. The enhanced turnover of PER by the ubiquitin-proteasome pathway [27] delays the accumulation and entry of PER into the nucleus through the Drosophila double time and its mammalian homolog,

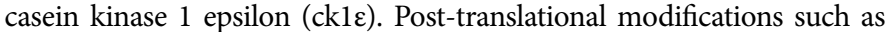
ubiquitination, SUMOylation and methylation [67-69] on the other hand, act to regulate the stability and function of the protein participating in the circadian clock. Recently, the trans-acting RNA-binding proteins (RBPs) like the polypyrimidine tract-binding protein and heterogeneous nuclear ribonucleoprotein $\mathrm{D}$ which bind to cis-acting elements in mammalian PER and CRY transcripts were found to get stabilized at different phases of the cycle $[70,71]$. When the length of the poly (A) tail is reduced, the efficiency of translation is found to be reduced because of the transcript destabilisation. This reduction in translational efficiency leads to the regulation of circadian clock and its output.

Mammalian Nocturnin, a circadian de-adenylase which is expressed in liver catalyses the poly(A) tail shortening [72,73], to control the metabolic rhythms. Other RBPs present in mammals and flies, such as LARK induce translation by putatively binding mRNAs of clock genes or clock output genes [44]. In addition, alternate splicing of the genes regulating clock which is sensitive to temperature is reported in flies, fungi and plants. They might found beneficial for allowing normal functioning of the clock for a wide temperature gradient. Besides, temperature sensitive alternate splicing has been found in flies, fungi and plants for controlling the clock function for a wide gradient of ambient temperature.

\section{Quest for rna interference in clock control}

The phenomena of RNAi which involves the expressional inhibition of the target gene through the destruction of the specific mRNA molecules can act as a potent tool for modifying the action of the circadian clock in an organism. There are usually two types of small ribonucleic acid (RNA) molecules - microRNA (miRNA) and small interfering RNA (siRNA) -important for achieving the aim of RNA interference. The miRNAs constitute a group of short, singlestranded, non-coding RNAs (nucleotide RNAs) of length 19-25 nt, that negatively regulate the expression of the gene by inhibiting the synthesis of target protein via repression of translation followed by breakdown of targets of miRNA. To achieve this target specificity, the miRNAs identify mRNAs which interact via base-pair complementarity with multiple sites within their 3' un-translated region (UTR). It is specifically the 5' miRNA region (called the "seed") which perfectly hybridizes with a 5 to
8 nucleotide stretch in the target 3' UTR and is necessary for initiating the communication.

\section{Rna induced core clock mechanism (Both RNAi and microRNA)}

The proposition of clock-controlled miRNAs which are expressed in a rhythm in multitude of tissues further raises a possibility of existence of a regulatory mechanism which is stringently controlled by the circadian clock. When the SCN was infused with the anti-sense oligonucleotide, intra-cerebroventricularly, it results in silencing the expression of miR-219 and produces a prolonged circadian locomotor activity under condition of constant darkness [74]. This clearly hints that miR-219 may be intricately related with modulating the speed of expression of the circadian clock. An over-expression of miR-219 in primary rat cortical neurons is found to suppress calcium influx which is induced by glutamate-NMDA and depolarization. NMDA has been portrayed in earlier in vivo and in vitro studies to undergo a negative regulation in presence of miR- 219 through the down-regulation of the calcium/calmodulin-dependent protein kinase II gamma sub-unit (CaMKII $\gamma$ ), which is a key component in NMDA receptor signalling cascade [75]. The bmal1 is also a potential target to the miR-219, present in the serum. The 3' UTR of bmall mRNA is a target to three miRNAs -miR-494, miR-152 and miR-142-3p of which the former two oscillate diurnally with peaks at mid-day while the latter one is constitutionally expressed all the time [76]. This shows that circulating miRNA finetunes the temporal expression of the peripheral circadian clock.

Studies by Nagel et al. in 2009, has enlisted miRNA -192/194 gene cluster to negatively regulate the expression of PER1, PER2 and PER3 [77]. While an over-expression of the same cluster [76] demonstrates a significant reduction of the circadian period length, which clearly shows that the miRNAs directly supervise the core component of the circadian clock.

An extensive study was made in unravelling the role of miRNAs in fine-tuning the core timing mechanism in Drosophila [78], wherein a knockdown in expression of the Dicer 1 gene in the oscillator cells were administered. This resulted in the dampening of the amplitude of the locomotor activity which concluded that the miRNAs play a vital role in maintaining of the circadian rhythm. Potential mRNA targets that were miRNA regulated were found to bind to AGO1. The clock components of CLK, VRI and CWO were found to undergo the repression by the miRNAin the tissue-specific drosha and pasha knockdown flies. The gene, bantam over-expressed in cell were found to extend the rhythm of the period locomotor activity by $2-3 \mathrm{hrs}$, proving that bantam indeed has a role in altering the molecular machinery within the central circadian pacemaker. Disruption studies of miRNA showed that miRNAs are essential to the sustenance of circadian rhythm and maintenance of a pace of the clock [78]. Thus, all the evidences clearly cite that the miRNAs functionally impact the circadian rhythm in flies and mammals by either targeting the clock gene directly or through indirect pathways that ultimately conduct inputs into the circadian clock.

\section{RNA Mediated Core Clock Input and Output}

The serial analysis of occupancy of the chromatin to screen for sites controlling the binding of the transcription factor have revealed miR132 as potential target for CREB protein which essentially resets the SCN clock [74]. The expression of miR-132, induced by light is found to be activated by the ERK/MAPK signalling pathway which proposes miR-132 to be a unit of the input pathway. Antisense knock down of the miR-132 establishes the miR-132 as a negative regulator of light 
entrainment. miR-132 is in fact, a negative transcription regulator of PER gene. This assay has clearly depicted that miR-132 is the key player in the regulation of the photic entrainment of the circadian clock in mammals, up-regulated in nocturnal light and down-regulated subsequently upon resetting the light effects on clock timing [74].

The in vitro 3' UTR reporter assay and protein estimation thereof elucidates the direct regulation of the miR-132 in case of the three genes that relate to chromatin (Mecp2, Jarid1a and Ep300) and two genes associated with translation (Btg2 and Paip2a). ChIP analysis has reported the abundance of MeCP2 binding sites in the PER1 and PER2 promoters which meant that an over-expression of MeCP2 leads to a CREB- dependent activation of PER1 and PER2. OTHER studies have shown that transcription induced by Clock/BMAL [79] is positively regulated by $\mathrm{p} 300$ while the histone $\mathrm{H} 3 \mathrm{~K} 4 \mathrm{me} 3$ methylation is negatively regulated by the histone demethylase JAR-ID1A [80]. Thus, in a nutshell, miR-132 has been shown to be involved in refinement of the circadian clock entrainment by coordinating the remodelling of chromatin with protein translation within the SCN. Thus, miRNA elicits a physiological action by regulating a subset of genes sharing the same biological function [81].

The miRNA-dependent regulation of the outputs of clock is mainly predominant in peripheral oscillators. The miRNA, miR-122 has been established to be responsible for the shaping of the gene expression in liver [82]. This miRNA was found to be at its peak in the morning but is relatively constant in case of the mature miR-122. The miR-122 bears promoter with two retinoic acid related orphan receptor responsive elements, constitutively elevated in liver deficient in Rev-ErbA [83]. miR-122 were found to effect the expression of $\operatorname{Ppar} \beta / \delta$ and PPARa co-activator, Amarcd1/baf60a [82]. A deadenylase enzyme, nocturnin, is considered to be the direct target of miR-122 since, a knockdown of miR-122 is found to increase the amplitude of nocturnin, with an increased expression. This raises a possibility that miRNA-mediated gene silencing is closely related to mRNA deadenylation [84]. The circadian oscillators in the vertebrate retina have shown the presence of miR-26a which is targeted and negatively regulates L-VGCCa1C, the l-type voltage gated Ca- channel $[85,86]$.

In Arabidopsis, a clock output process of photoperiodic flowering is regulated by miRNA. GIGANTEA and CONSTANS proteins are involved in inducing the expression of floral integrators, which in turn helps in photoperiodic flowering [87]. The miR-172 is involved in photoperiodic flowering by inducing the cleavage of the TOE1 mRNA [88]. This is one of the pioneer studies, where it was shown that miRNAs are involved in photoperiodic control of flowering time and thus involved in circadian rhythm of higher plants.

\section{Future prospective for relation with the long non coding rnas}

lncRNAs are non-protein transcripts having a length more than 200 nucleotides [89], which are found to regulate the vertebrate circadian systems significantly. A recent study has shown 112 lncRNAs to have a variable expression between day/night within the pineal gland of rats [90]. Detailed study of these eight highly rhythmic lncRNA has highlighted the role of neurons from the SCN as well as external stimuli like light exposure onto periodicity and amplitude of circadian lncRNAs. A cyclic expression of ncRNAs like NATs, lncRNAs and miRNAs result in chromatin modifications in the liver in a rhythm [91]. PER2, which is a circadian oscillator itself, is under the control of an antisense lncRNA, PER2. The Prader-Willi syndrome (PWS), which is a genetic disorder controlled by the CNS, affects the circadian rhythm. It is found to be linked to a PWS- associated
IncRNA called 116G, which causes metabolism deregulation and obesity development upon mutation. An lncRNA with the remaining $116 \mathrm{G}$ is formed in the mice brain after splicing, which elicits a physiological circadian rhythm after binding to the transcriptional activator, RBBP5. Mutation in this lncRNA, 116HG result in a deregulated expression of diurnally expressed circadian genes, Clock, Cry1, and PER2 in the CNS [92]. The lncRNA, HULC which is over-expressed in hepatocellular carcinoma (HCC), interrupts the circadian rhythm of hepatoma cells increasing the incidence of hepato carcinogenesis through the alteration of the expression pattern of the circadian oscillator, CLOCK [93]. Studies by Dr. Liu and his team on the bread mold, Neurospora crassa, has revealed new findings about a long non-coding RNA, qrf, which controls the expression of a clock gene named frequency (frq) [94]. The lncRNA is a RNA molecule that is complementary, or antisense, to frq. Contrary to other RNA molecules, qrf itself does not encode a protein, but in turn regulates the amount of protein, frq synthesized. This qrf RNA is produced in presence of light and once produced; modulates the production of the frq protein. Henceforth, it resets the circadian clock in a lightdependent manner. The gene frq is found to regulate the reverse phase operation of the clock gene by blocking the qrf production by frq. Absence of $q r f$, therefore results in disrupted circadian rhythm which highlights the crucial role of the long non-coding RNA is crucial for the functioning of the clock gene. These excerpts clearly highlight the importance of lncRNA in maintaining the circadian rhythm across different species.

\section{Conclusion}

This review has exposed the various cues modulating the initiation and sustenance of a fine-tuned biological rhythm. The cues include extensive networks which are intercellular as well as intracellular in origin. The chief players in this modulation, in the form of genes, transcription factors or abiotic factors, even though are variable across a plethora of species; convey the unified essence of synchronization to elicit a response to the most preferable cue. In this way, the organism makes apt responses to the variable environmental situations, obliterating the uncanny, irrelevant signals that might otherwise hoax the physiological processes. The biological rhythms in different organisms have evolutionarily evolved for the prime reason of acclimatizing the organism to changed environmental conditions. This aspect seems to be highly appreciated in realising how beautifully the organisms' cellular machinery assists it in abrogating the undesired cues. It is therefore evolutionarily preferred not only among lower grade of organisms but also among the human species. The current scenario exposes us to a multitude of cues which act as disruptors of the normal rhythm. Once such a situation is realised, there arises a constellation of lifestyle disorders, which are due to disrupted feeding and sleeping pattern. If the RNAi technology is harnessed in correcting these malfunctions, an effective remedy devoid of any side-effects can be achieved.

Exhaustive exemplification of the subsets of miRNAs and noncoding RNAs involved in the maintenance of biological clock has opened a new avenue for circadian researchers all over the world to search for further putative pathways and genes that can promise to be a better target to developing medications for ailments involving a malfunctioned circadian rhythm, including the sleeping disorders, development of insulin resistance and cancer. Although present contributions have been realised unprecedentedly, further advances are yet to be achieved in this newly emerged and unexplored world of circadian rhythm which is in close communion with the silencing of gene. 


\section{Competing Interests}

The authors declare that they do not have any competing interests.

\section{Authors' Contributions}

PD wrote the initial write up. UB designed the review and UB and MPB contributed the final write.

Nirav Thakkar gave the cosmetic touch of the figures and Anisha Pal helped in early write and figures drawing.

\section{Acknowledgement}

The work was supported by an HFSP Young Investigator Grant (RGY020), CSIR network project (B.Sc 0108 and B.Sc 0121) awarded to UB. Welcome Trus International Fellowship awarded to MPB (GAP0065).

\section{References}

1. Panda S, Hogenesch JB, Kay SA (2002) Circadian rhythms from flies to human Nature 417: 329-335.

2. Mehta N, Cheng HY (2013) Micro-managing the circadian clock: The role of microRNAs in biological time keeping. J Mol Biol 425: 3609-3624.

3. Konopka RJ, Benzer S (1971) Clock mutants of Drosophila melanogaster. Proc Natl Acad Sci USA 68: 2112-2116

4. Krishnan B, Dryer SE, Hardin PE (1999) Circadian rhythms in olfactory responses of Drosophila melanogaster. Nature 400: 375-378.

5. Hari Dass TMS, Sharma VK (2008) Egg-laying rhythm in Drosophila melanogaster. J Genet 87: 495-504.

6. Fujii S, Krishnan P, Hardin P, Amrein H (2007) Nocturnal male sex drive in Drosophila. Curr Biol 17: 244-251.

7. Fujii S, Amrein H (2010) Ventral lateral and DN1 clock neurons mediate distinct properties of male sex drive rhythm in Drosophila. Proceedings of the National Academy of Sciences. 107:10590-10595.

8. Chatterjee A, Tanoue S, Houl JH, Hardin PE (2010) Regulation of gustatory physiology and appetitive behavior by the Drosophila circadian clock. Curr Bio 20: $300-309$

9. Lyons LC, Roman G (2008) Circadian modulation of short-term memory in Drosophila. Learn Mem 16: 19-27.

10. Pittendrigh CS (1967) Circadian systems. I. The driving oscillation and its assay in Drosophila pseudoobscura. Proc Natl Acad Sci USA 58: 1762-1767.

11. Hendricks JC, Finn SM, Panckeri KA, Chavkin J, Williams JA, et al. (2000) Rest in Drosophila is a sleep-like state. Neuron 25: 129-138.

12. Shaw PJ, Cirelli C, Greenspan RJ, Tononi G (2000) Correlates of sleep and waking in Drosophila melanogaster. Science 287: 1834-1837.

13. Bargiello TA, Young MW (1984) Molecular genetics of a biological clock in Drosophila. Proc Natl Acad Sci USA 81: 2142-2146.

14. Bargiello TA, Jackson FR, Young MW (1984) Restoration of circadian behavioural rhythms by gene transfer in Drosophila. Nature 312: 752-754.

15. Sawyer LA, Hennessy JM, Peixoto AA, Rosato E, Parkinson H, et al. (1997) Natural variation in a Drosophila clock gene and temperature compensation. Science 278: 2117-2120.

16. Hardin PE (2011) Molecular genetic analysis of circadian time keeping in Drosophila. Adv Genet 74: 141-173.

17. Nambu JR, Lewis JO, Wharton KA, Crews ST (1991). The Drosophila singleminded gene encodes a helix-loop-helix protein that acts as a master regulator of CNS midline development. Cell. 67:1157-1167.

18. Huang ZJ, Edery I, Rosbash M (1993) PAS is a dimerization domain common to Drosophila period and several transcription factors. Nature 364: 259-262.

19. Hardin PE, Hall JC, Rosbash M (1990) Feedback of the Drosophila period gene product on circadian cycling of its messenger RNA levels. Nature 343: 536-540.

20. Siwicki KK, Eastman C, Petersen G, Rosbash M, Hall JC (1988) Antibodies to the period gene product of Drosophila reveal diverse tissue distribution and rhythmic changes in the visual system. Neuron 1: 141-150.

21. Hardin PE, Hall JC, Rosbash M (1992) Circadian oscillations in period gene mRNA levels are transcriptionally regulated. Proc Natl Acad Sci USA 89: 1171111715.
22. Zeng $\mathrm{H}$, Hardin PE, Rosbash $M$ (1994) Constitutive overexpression of the Drosophila period protein inhibits period mRNA cycling. EMBO J 13: 3590-3598.

23. Sehgal A, Price JL, Man B, Young MW (1994) Loss of circadian behavioral rhythms and per RNA oscillations in the Drosophila mutant timeless. Science 263: 1603-1606.

24. Allada R, White NE, So WV, Hall JC, Rosbash M (1998). A mutant Drosophila homolog of mammalian Clock disrupts circadian rhythms and transcription of period and timeless. Cell. 93:791-804.

25. Rutila JE, Suri V, Le M, So WV, Rosbash M, et al. (1998) Cycle is a second bHLH-PAS clock protein essential for circadian rhythmicity and transcription of Drosophila period and timeless. Cell 93: 805-814.

26. Kloss B, Price JL, Saez L, Blau J, Rothenfluh A, et al (1998). The Drosophila clock gene double-time encodes a protein closely related to human casein kinase le. Cell. 94: 97-107.

27. Price JL, Blau J, Rothenfluh A, Abodeely M, Kloss B, et al. (1998) Double-time is a novel Drosophila clock gene that regulates period protein accumulation. Cell 94: 83-95.

28. Martinek S, Inonog S, Manoukian AS, Young MW (2001) A role for the segment polarity gene shaggy/GSK-3 in the Drosophila circadian clock. Cell 105: 769 779

29. Akten B, Jauch E, Genova GK, Kim EY, Edery I, et al. (2003) A role for CK2 in the Drosophila circadian oscillator. Nat Neurosci 6: 251-257.

30. Lin JM, Kilman VL, Keegan K, Paddock B, Emery-Le M, et al. (2002) A role for casein kinase 2 alpha in the Drosophila circadian clock. Nature 420: 816-820.

31. Stanewsky R, Kaneko M, Emery P, Beretta B, Wager-Smith K, et al. (1998) The cryb mutation identifies cryptochrome as a circadian photoreceptor in Drosophila. Cell. 95: 681-692.

32. Gekakis N, Saez L, Delahaye-Brown AM, Myers MP, Sehgal A, et al. (1995) Isolation of time less by PER protein interaction: defective interaction between timeless protein and long-period mutant PERL. Science. 270: 811-815.

33. Hao H, Allen DL, Hardin PE (1997). A circadian enhancer mediates PERdependent mRNA cycling in Drosophila melanogaster. Molecular and cellular biology. 17: 3687-3693

34. King DP, Vitaterna MH, Chang AM, Dove WF, Pinto LH, et al (1997). The mouse clock mutation behaves as an antimorph and maps within the $\mathrm{W} 19 \mathrm{H}$ deletion, distal of Kit. Genetics.146:1049-1060

35. King DP, Zhao Y, Sangoram AM, Wilsbacher LD, Tanaka M, et al. (1997) Positional cloning of the mouse circadian clockgene. Cell. 89:641-653.

36. Vitaterna MH, King DP, Chang AM, Kornhauser JM, Lowrey PL, et al. (1994) Mutagenesis and mapping of a mouse gene, Clock, essential for circadian behavior. Science 264: 719-725.

37. Darlington TK, Wager-Smith K, Ceriani MF, Staknis D, Gekakis N, et al. (1998) Closing the circadian loop: Clock-induced transcription of its own inhibitors PER and TIM. Science 280: 1599-1603.

38. Bae K, Lee C, Sidote D, Chuang KY, Edery I (1998). Circadian regulation of a Drosophila homolog of the mammalian Clock gene: PER and TIM function as positive regulators. Molecular and cellular biology. 18: 6142-6151.

39. Glossop NR, Lyons LC, Hardin PE (1999) Interlocked feedback loops within the Drosophila circadian oscillator. Science 286: 766-768.

40. Cyran SA, Buchsbaum AM, Reddy KL, Lin MC, Glossop NR, et al. (2003) vrille, Pdp1, and dClock form a second feedback loop in the Drosophila circadian clock. Cell 112: 329-341.

41. Glossop NR, Houl JH, Zheng H, Ng FS, Dudek SM, et al. (2003) Vrille feeds back to control circadian transcription of Clock in the Drosophila circadian oscillator. Neuron 37: 249-261.

42. Zheng X, Koh K, Sowcik M, Smith CJ, Chen D, et al. (2009) An isoform-specific mutant reveals a role of PDP1 epsilon in the circadian oscillator. J Neurosci 29 10920-10927.

43. Kadener S, Stoleru D, McDonald M, Nawathean P, Rosbash M (2007) Clockwork Orange is a transcriptional repressor and a new Drosophila circadian pacemaker component. Genes Dev 21: 1675-1686.

44. Kojima S, Matsumoto K, Hirose M, Shimada M, Nagano M, et al. (2007) Lark activates posttranscriptional expression of an essential mammalian clock protein Period1. Proceedings of the National Academy of Sciences. 104:1859-1864. 
45. Lim C, Chung BY, Pitman JL, McGill JJ, Pradhan S, et al (2007). Clockwork orange encodes a transcriptional repressor important for circadian-clock amplitude in Drosophila. Current biology; 17:1082-1089.

46. Matsumoto A, Ukai-Tadenuma M, Yamada RG, Houl J, Uno KD, et al. (2007) A functional genomics strategy reveals clockwork orange as a transcriptional regulator in the Drosophila circadian clock. Genes \& development.21:1687-1700.

47. Chiu JC, Vanselow JT, Kramer A, Edery I (2008). The phospho-occupancy of an atypical SLIMB-binding site on Period that is phosphorylated by double time controls the pace of the clock. Genes \& development. 22:1758-1772.

48. Kivimae S, Saez L, Young MW (2008) Activating PER repressor through a DBTdirected phosphorylation switch. PLoS Biol 6: e183.

49. Lin JM, Schroeder A, Allada R (2005) In vivo circadian function of casein kinase 2 phosphorylation sites in Drosophila period. J Neurosci 25: 11175-11183.

50. Chiu JC, Ko HW, Edery I (2011) NEMO/NLK phosphorylates period to initiate a time-delay phosphorylation circuit that sets circadian clock speed. Cell 145: 357-370

51. Yu W, Houl JH, Hardin PE (2011) NEMO kinase contributes to core period determination by slowing the pace of the Drosophila circadian oscillator. Curr Biol 21: 756-761.

52. Fang Y, Sathyanarayanan S, Sehgal A (2007) Post-translational regulation of the Drosophila circadian clock requires protein phosphatase 1 (PP1). Genes \& development. 21: 1506-1518.

53. Sathyanarayanan S, Zheng X, Xiao R, Sehgal A (2004) Posttranslational regulation of Drosophila PERIOD protein by protein phosphatase 2A. Cell 116: 603-615.

54. Nawathean P, Rosbash M (2004) The double time and CKII kinases collaborate to potentiate Drosophila PER transcriptional repressor activity. Mol Cell 13: 213-223.

55. Kim EY, Edery I (2006) Balance between DBT/CKle kinase and protein phosphatase activities regulate phosphorylation and stability of Drosophila CLOCK protein. Proceedings of the National Academy of Sciences. 103: 61786183.

56. Yu W, Zheng H, Houl JH, Dauwalder B, Hardin PE (2006). PER-dependent rhythms in CLK phosphorylation and E-box binding regulate circadian transcription. Genes \& development. 20:723-733.

57. Yu W, Zheng H, Price JL, Hardin PE (2009). Double time plays a non-catalytic role to mediate CLOCK phosphorylation and repress CLOCK-dependent transcription within the Drosophila circadian clock. Molecular and cellular biology.29: 1452-1458.

58. Naidoo N, Song W, Hunter-Ensor M, Sehgal A (1999) A role for the proteasome in the light response of the timeless clock protein. Science 285: 1737-1741.

59. Emery P, So WV, Kaneko M, Hall JC, Rosbash M (1998) CRY, a Drosophila clock and light-regulated cryptochrome, is a major contributor to circadian rhythm resetting and photosensitivity. Cell 95: 669-679.

60. Ahmad M, Cashmore AR (1993) HY4 gene of $A$. thaliana encodes a protein with characteristics of a blue-light photoreceptor. Nature 366: 162-166.

61. Lin C, Yang H, Guo H, Mockler T, Chen J, et al. (1998) Enhancement of bluelight sensitivity of Arabidopsis seedlings by a blue light receptor cryptochrome 2. Proc Natl Acad Sci USA 95: 2686-2690.

62. Koh K, Zheng X, Sehgal A (2006) Jetlag resets the Drosophila circadian clock by promoting light-induced degradation of timeless. Science 312: 1809-1812.

63. Peschel N, Veleri S, Stanewsky R (2006) Veela defines a molecular link between Cryptochrome and Timeless in the light-input pathway to Drosophila's circadian clock. Proceedings of the National Academy of Sciences.103: 1731317318

64. Peschel N, Chen KF, Szabo G, Stanewsky R (2009) Light-dependent interactions between the Drosophila circadian clock factors cryptochrome, jetlag, and timeless. Current biology. 19: 241-247.

65. Hogenesch JB, Herzog ED (2011) Intracellular and intercellular processes determine robustness of the circadian clock. FEBS Lett 585: 1427-1434.

66. Johnson CH, Stewart PL, Egli M (2011) The cyanobacterial circadian system: from biophysics to bioevolution. Annu Rev Biophys 40: 143-167.

67. Cardone L, Hirayama J, Giordano F, Tamaru T, Palvimo JJ, et al. (2005) Circadian clock control by sumoylation of BMAL1. Science 309: 1390-1394.
68. Busino L, Bassermann F, Maiolica A, Lee C, Nolan PM, et al. (2007) SCFFbxl3 controls the oscillation of the circadian clock by directing the degradation of cryptochrome proteins. Science 316: 900-904.

69. Katada S, Sassone-Corsi P (2010) The histone methyltransferase MLL1 permits the oscillation of circadian gene expression. Nat Struct Mol Biol 17 1414-1421.

70. Woo KC, Kim TD, Lee KH, Kim DY, Kim W, et al. (2009) Mouse period 2 mRNA circadian oscillation is modulated by PTB-mediated rhythmic mRNA degradation. Nucleic Acids Res 37: 26-37.

71. Woo KC, Ha DC, Lee KH, Kim DY, Kim TD, et al. (2010) Circadian amplitude of cryptochrome 1 is modulated by mRNA stability regulation via cytoplasmic hnRNP D oscillation. Mol Cell Biol 30: 197-205.

72. Baggs JE, Green CB (2003) Nocturnin, a deadenylase in Xenopus laevis retina: a mechanism for post-transcriptional control of circadian-related mRNA. Curr Biol 13: 189-198.

73. Green CB, Douris N, Kojima S, Strayer CA, Fogerty J, et al (2007). Loss of Nocturnin, a circadian deadenylase, confers resistance to hepatic steatosis and diet-induced obesity. Proceedings of the National Academy of Sciences. 104 9888-9983.

74. Cheng HY, Papp JW, Varlamova O, Dziema H, Russell B, et al. (2007) microRNA modulation of circadian-clock period and entrainment. Neuron 54 813-829.

75. Kocerha J, Faghihi MA, Lopez-Toledano MA, Huang J, Ramsey AJ, et al (2009). MicroRNA-219 modulates NMDA receptor-mediated neurobehavioral dysfunction. Proceedings of the National Academy of Sciences. 106: 35073512.

76. Shende VR, Goldrick MM, Ramani S, Earnest DJ (2011) Expression and rhythmic modulation of circulating microRNAs targeting the clock gene Bmal1 in mice. PLoS One 6: e22586.

77. Nagel R, Clijsters L, Agami R (2009) The miRNA-192/194 cluster regulates the Period gene family and the circadian clock. FEBS J 276: 5447-5455.

78. Kadener S, Menet JS, Sugino K, Horwich MD, Weissbein U, et al. (2009) A role for microRNAs in the Drosophila circadian clock. Genes Dev 23: 2179-2191.

79. Etchegaray JP, Lee C, Wade PA, Reppert SM (2003) Rhythmic histone acetylation underlies transcription in the mammalian circadian clock. Nature. 421:177-182.

80. Ripperger JA, Schibler U (2006) Rhythmic Clock-BMAL1 binding to multiple E-box motifs drives circadian Dbp transcription and chromatin transitions. Nat Genet 38: 369-374.

81. Alvarez-Saavedra M, Antoun G, Yanagiya A, Oliva-Hernandez R, CornejoPalma D, et al. (2011) miRNA-132 orchestrates chromatin remodeling and translational control of the circadian clock. Human molecular genetics 20:731 751.

82. Gatfield D, Le Martelot G, Vejnar CE, Gerlach D, Schaad O, et al. (2009) Integration of microRNA miR-122 in hepatic circadian gene expression. Genes Dev 23: 1313-1326.

83. Esau C, Davis S, Murray SF, Yu XX, Pandey SK, et al. (2006) miR-122 regulation of lipid metabolism revealed by in vivo antisense targeting. Cell Metab 3: 87-98.

84. Kojima S, Gatfield D, Esau CC, Green CB (2010) MicroRNA-122 modulates the rhythmic expression profile of the circadian deadenylase Nocturnin in mouse liver. PLoS One 5: e11264.

85. Ko ML, Liu Y, Dryer SE, Ko GYP. The expression of L-type voltage-gated calcium channels in retinal photoreceptors is under circadian control. Journal of neurochemistry 103: 784-92.

86. Ko ML, Jian K, Shi L, Ko GY (2009) Phosphatidylinositol 3 kinase-Akt signaling serves as a circadian output in the retina. J Neurochem 108: 1607-1620.

87. Yanovsky MJ, Kay SA (2003) Living by the calendar: how plants know when to flower. Nat Rev Mol Cell Biol 4: 265-275.

88. Jung JH, Seo YH, Seo PJ, Reyes JL, Yun J, et al. The Gigantea-regulated microRNA172 mediates photoperiodic flowering independent of constants in Arabidopsis. The Plant Cell 19: 2736-2748.

89. Perkel JM (2013) Visiting "noncodarnia”. Biotechniques 54: 301, 303-304.

90. Coon SL, Munson PJ, Cherukuri PF, Sugden D, Rath MF, et al. (2012) Circadian changes in long noncoding RNAs in the pineal gland. Proc Natl Acad Sci USA 109: 13319-13324. 
Citation: Bhadra U, Das P, Pal-Bhadra M (2016) RNA Interference (RNAi) as a metronome of the circadian cadence. Mol Biol 5: 166. doi:10.4172/21689547.1000166

91. Vollmers C, Schmitz RJ, Nathanson J, Yeo G, Ecker JR, et al. (2012) Circadian oscillations of protein-coding and regulatory RNAs in a highly dynamic mammalian liver epigenome. Cell Metab 16: 833-845.

92. Powell WT, Coulson RL, Crary FK, Wong SS, Ach RA, et al. A Prader-Willi locus IncRNA cloud modulates diurnal genes and energy expenditure. Human molecular genetics 22: 4318-4328.
93. Cui M, Zheng M, Sun B, Wang $Y$, Ye $L$, et al. A long noncoding RNA perturbs the circadian rhythm of hepatoma cells to facilitate hepatocarcinogenesis. Neoplasia 17:79-88.

94. Jinhu G, Yi L (2010) Molecular mechanism of the Neurospora circadian oscillator. Protein Cell 1: 331-341. 